\title{
Letter to the editor Re: Singh I et al.: growing skull fractures: guidelines for early diagnosis and surgical management (2016)
}

\author{
Paul Steinbok ${ }^{1}$
}

Received: 29 August 2016 / Accepted: 1 September 2016 / Published online: 9 September 2016

(C) Springer-Verlag Berlin Heidelberg 2016

Dear Editor,

I wish to commend Singh et al. [1] for their interesting paper on early diagnosis and management of growing skull fractures. I agree with the authors that those at risk of developing a growing skull fracture include young children with a diastatic fracture and an underlying brain contusion, which were the criteria for inclusion of the patients in their study for evaluation of a possible growing skull fracture. In this group of patients, the authors have shown that with MRI it is possible to identify an underlying dural laceration. Since there needs to be a dural laceration for a growing skull fracture to evolve, identification of such a dural laceration identifies a population at highest risk of developing a growing skull fracture. Furthermore, I agree with the authors that if there is no dural laceration underlying a diastatic fracture, there is no risk of a growing skull fracture developing.

However, we do not know what percentage of children in their cohort with an identified dural laceration would have gone on to develop a growing skull fracture without intervention. It may well be that some patients in this group with a dural laceration would not have developed a growing skull fracture. I have not reviewed this specifically, but it is my recollection that the vast minority of young children with a diastatic fracture and underlying brain contusion that I have managed have not developed a growing skull fracture. None have had MRIs to look for a dural laceration, so I do not know how many, if any, of these children did have an underlying dural laceration, but I suspect that some did.

Thus, I have problems with recommending an early operation, as the authors did, for all of the patients in whom a dural

Paul Steinbok

psteinbok@cw.bc.ca

1 Division of Neurosurgery, BC Children's Hospital and University of British Columbia, 4480 Oak St., Room K3-159, Vancouver, BC V6h 3V4, Canada laceration was identified. As the authors noted, this early surgery was complicated by much inflammatory response and a significant amount of blood loss, leading to blood transfusion in over $60 \%$.

My approach to the patients at risk of developing a growing skull fracture is simply to reassess at about 4 weeks after trauma to look for clinical evidence, suggesting a growing skull fracture, such as a pulsatile mass or bony defect at the site of the known diastatic fracture. A skull radiograph will usually indicate if the fracture edges have separated since the original trauma. For patients with no clinical or skull radiographic evidence of a growing skull fracture at this 4-week visit, no further follow-up or radiologic investigations are done. If there are equivocal findings, I would reassess after another 1 to 2 months. For those with evidence of an early growing skull fracture (at 4 weeks), surgery is offered, and a MRI is done prior to that surgery. With such a protocol, I think that patients with a growing skull fracture have this identified and treated in a timely manner. Those high risk patients who do not develop a growing skull fracture are spared an operation, compared to the management protocol advocated by Singh et al. [1]. Furthermore, compared to Singh et al.'s protocol, my protocol reduces the number of MRIs, which, in this population, usually require general anesthesia. This has the added benefit of reducing costs to the health care system.

\section{Compliance with ethical standards}

Conflicts of interest There are no conflicts of interest.

\section{References}

1. Singh I, Rohilla S, Siddiqui SA, Kumar P (2016) Growing skull fractures: guidelines for early diagnosis and surgical management. Child's nervous system: ChNS: official journal of the International Society for Pediatric Neurosurgery 32:1117-1122 